\title{
Cartão Nacional de Saúde: Avaliação da Confiabilidade em Bases de Dados Codificadas da Oncologia
}

doi: https://doi.org/10.32635/2176-9745.RBC.2019v65n2.337

\author{
National Health Card: Assessment of Reliability of Oncology Encoded Databases \\ Tarjeta Nacional de Salud: Evaluación de la Conformidad en Bases de Datos de la Oncología Codificadas
}

Adriana Tavares de Moraes Atty'; Jeane Glaucia Tomazelli²; Maria Beatriz Kneipp Dias ${ }^{3}$; Caroline Madalena Ribeiro ${ }^{4}$; Arn Migowski'; Neilane Bertoni ${ }^{6}$

Resumo

Introdução: O Cartão Nacional de Saúde (CNS) foi criado para ser um instrumento de identificação individualizada do cidadão. Nas bases de dados disponibilizadas pelo Departamento de Informática do Sistema Único de Saúde (DATASUS), a variável CNS é codificada. Objetivo: Verificar se a variável CNS, codificada nas bases de dados do DATASUS, pode ser utilizada como identificador unívoco do usuário. Método: Realizou-se relacionamento determinístico entre a base de Autorizaçóes de Procedimentos de Alta Complexidade em Oncologia (Apac-onco) de quimioterapia e radioterapia do DATASUS e as de um Hospital referência em oncologia no Estado do Rio de Janeiro, entre 2010 a 2016. Resultados Na base de dados de quimioterapia, 2,83\% dos CNS tinham mais de uma codificaçáo, e, na de radioterapia, 0,55\% também apresentavam mais de uma codificaçáo. Consequentemente, a utilização da CNS codificada excedeu a contagem de 45,5\% das 77 CID no banco de quimioterapia e 20,2\% das 84 CID no de radioterapia. Conclusáo: No formato atual, o CNS codificado não garante identificação unívoca dos usuários, dificultando o acompanhamento dos procedimentos, a estimativa de custos de tratamento, a identificação de barreiras de acesso e o planejamento da organizaçáo da rede de atenção à saúde.

Palavras-chave: Confidencialidade; Sistemas de Informação; Sistema Único de Saúde; Bases de Dados como Assunto.

\begin{abstract}
Introduction: The National Health Card (CNS) was created to be an instrument of individual identification of the citizen. In the databases provided by DATASUS, the variable CNS is encoded. Objective: To verify if the encoded variable of the CNS in public databases provided by DATASUS can be used as a unique identifier of the patient. Method: It was conducted a deterministic linkage between the High Complexity Procedures in Oncology (Apac-onco) database of chemotherapy and radiotherapy, provided by DATASUS, and the databases of an oncology reference hospital located in the State of Rio de Janeiro, considering data from 2010 to 2016. Results: In the database of chemotherapy, $2.83 \%$ of the CNSs had more than one encoding and, in radiotherapys, $0.55 \%$ similarly. Consequently, the utilization of the CNS encoded exceeded the count of $45.5 \%$ of the 77 CID of the chemotherapy database and $20.2 \%$ of the 84 CID of radiotherapy. Conclusion: In the current format, the CNS encoded does not ensure a univocal identification of SUS patients, making it difficult to follow up the procedures, to estimate the treatment costs, identify barriers to access and plan the health care system organization.

Key words: Confidentiality; Information Systems; Unified Health System; Databases as Topic.
\end{abstract}

Resumen

Introducción: La Tarjeta Nacional de Salud (CNS) fue creada para ser un instrumento de identificación individualizada del ciudadano. En las bases de datos proporcionadas por DATASUS, la variable CNS está codificada. Objetivo: Verificar si la variable CNS codificada en las bases de datos de DATASUS puede ser utilizada como identificador unívoco del usuario. Método: Se realizó una relación determinista entre la base de Autorizaciones de Procedimientos de Alta Complejidad en Oncología (Apaconco) de quimioterapia y radioterapia, de DATASUS, y las de un Hospital referencia en oncología en el Estado de Río de Janeiro, entre 2010 a 2016. Resultados: En la base de datos de quimioterapia, 2,83\% de los CNS tenía más de una codificación, y en radioterapia, el 0,55\% también tenía más de una codificación. En consecuencia, la utilización de la CNS codificada sobreestimó el conteo del 45,5\% de las 77 CID en el banco de quimioterapia y el 20,2\% de las 84 CID en la radioterapia. Conclusión: En el formato actual, el CNS codificado no garantiza identificación unívoca de los usuarios, dificultando el seguimiento de los procedimientos, la estimación de costos de tratamiento, la identificación de barreras de acceso y la planificación de la organización de la red de atención a la salud.

Palabras clave: Confidencialidad; Sistemas de Información; Sistema Único de Salud; Bases de Datos como Asunto.

\footnotetext{
${ }^{1}$ Instituto Nacional de Câncer José Alencar Gomes da Silva (INCA). Rio de Janeiro (RJ), Brasil. Orcid iD: https://orcid.org/0000-0003-2271-746X ${ }^{2}$ INCA. Rio de Janeiro (RJ), Brasil. Orcid iD: https://orcid.org/0000-0002-2472-3444

${ }^{3}$ INCA. Rio de Janeiro (RJ), Brasil. Orcid iD: https://orcid.org/0000-0002-5847-9830

${ }^{4}$ INCA. Rio de Janeiro (RJ), Brasil. Orcid iD: https://orcid.org/0000-0003-2690-5791

${ }^{5}$ INCA. Rio de Janeiro (RJ), Brasil. Orcid iD: https://orcid.org/0000-0002-4861-2319

${ }^{6}$ INCA. Rio de Janeiro (RJ), Brasil. Orcid iD: https://orcid.org/0000-0002-2539-9965

Endereço para correspondência: Adriana Tavares de Moraes Atty. Rua Marquês de Pombal, 125, 70 andar - Centro. Rio de Janeiro (RJ), Brasil. CEP 22.230-240.

E-mail: aatty@inca.gov.br
} 


\section{INTRODUÇÃO}

O Cartão Nacional de Saúde (CNS) foi criado para ser um instrumento de identificação individualizada do cidadáo para permitir o acompanhamento de sua trajetória no Sistema Único de Saúde (SUS), reorientando o modelo de atenção à saúde ${ }^{1-3}$. Sua adoçáo pelos Sistemas de Informação em Saúde (SIS) possibilitaria conhecer o perfil dos usuários, os entraves nas redes de serviços de saúde, a cobertura do SUS, além das referências entre os entes federados ${ }^{2}$.

A implementação do CNS tem sido gradativa ao longo do tempo. Instituído pela Portaria n. ${ }^{\circ} 1.560$ de $2002^{4}$, em $2004^{5}$, passou a ser obrigatório em procedimentos ambulatoriais (Autorizaçóes de Procedimentos de Alta Complexidade - Apac) e hospitalares (Autorizaçôes de Internações Hospitalares - AIH) que necessitassem de autorização prévia. Já a regulamentação ocorreu em 2011, cabendo ao Departamento de Informática do Sistema Único de Saúde (DATASUS) realizar a verificação das informaçóes cadastradas nos diferentes sistemas, compatibilizando-as com a base de dados do CNS, identificando duplicidades e inconsistências cadastrais ${ }^{3}$.

A fim de promover a organizaçáo de uma rede de serviços regionalizada e hierarquizada, foram regulamentados diversos padróes de interoperabilidade e de informação em saúde no âmbito dos sistemas do SUS, da saúde suplementar e de sistemas privados para a integração de sistemas de saúde, legados ou novos. Com dados de qualidade e identificação única de CNS por indivíduo, seria possível relacionar as informaçóes dos usuários do SUS em diferentes sistemas de informação, visando à implementação de um Registro Eletrônico de Saúde (RES) nacional e longitudinal ${ }^{6}$.

A qualificação da base do CNS prevê um processo de padronização e correção de dados, que inclui o relacionamento com o banco de dados da Receita Federal ${ }^{7}$. Uma vez aplicados os critérios de qualidade, é atribuído um número "master" ao indivíduo, e a esse número são agregados os demais CNS que ele eventualmente possua. Esse processo, denominado "higienização da base de dados", é contínuo e realizado exclusivamente pelo DATASUS.

Informaçôes sobre os atendimentos prestados no SUS estão disponibilizadas no sítio eletrônico do DATASUS (www.datasus.gov.br), sem restriçôes de acesso, mas resguardando o direito à privacidade e ao sigilo da identificação dos usuários, por meio da codificação da variável $\mathrm{CNS}^{7,8}$. Assim, o poder público garante a proteção da informação, sua disponibilidade, autenticidade e integralidade, ao mesmo tempo em que promove a divulgação de informaçôes de interesse coletivo por ele produzidas ou custodiadas?.
Vislumbrar a possibilidade de acompanhar o percurso terapêutico do usuário, avaliar a qualidade da assistência e aprimorar a rede de atenção à saúde, por intermédio dos vários SIS de acesso livre, seria uma conquista para gestão do SUS. Assim, o objetivo deste estudo foi verificar se a variável codificada referente ao CNS, disponível em bases de dados do DATASUS, de fato pode ser utilizada como identificador unívoco de usuários em estudos de dados individualizados.

\section{MÉTODO}

Foi realizado relacionamento determinístico entre a base de dados das Apac-onco, disponibilizada na Internet pelo DATASUS, e a base de um Hospital de referência, o Instituto Nacional de Câncer José Alencar Gomes da Silva (INCA), habilitado em oncologia no Estado do Rio de Janeiro. Os dados referem-se aos procedimentos de quimioterapia e radioterapia realizados entre 2010 a 2016. O relacionamento foi feito pela variável 'número de autorização' (número da Apac).

A base de dados Apac-onco disponibilizada pelo DATASUS possui a variável "Cartão Nacional de Saúde Codificada”, impossibilitando a identificação do usuário. Foram selecionadas apenas as autorizaçôes de quimioterapia e radioterapia iniciais, Apac tipo 1, considerando que estas representam a primeira autorizaçáo do paciente dentro do período de validade da Apac. Utilizaram-se também as variáveis: número da autorização das Apac, CNS codificado, sexo, o código da Classificação Estatística Internacional de Doenças e Problemas Relacionados à Saúde (CID-10) e o código do estabelecimento de saúde registrado no Cadastro Nacional de Estabelecimentos de Saúde (Cnes do estabelecimento). O download da base Apac-onco foi realizado em 23 de março de 2018.

A base de dados com a produção das Apac-onco obtida do hospital de referência era identificada com o número do cartáo SUS (CNS), nome do paciente, sexo, data de nascimento, número da autorização das Apac e Cnes do estabelecimento de saúde, além de também possuir outras informaçôes clínicas, como a CID-10 e o ano de tratamento.

Para avaliar a unicidade das codificações atribuídas pelo DATASUS aos CNS, foram verificadas as possíveis situaçóes: (a) ocorrência de mais de um número de CNS com uma mesma codificaçáo; (b) ocorrência de mais de um código para um mesmo número de CNS. Também foi avaliado se, para uma mesma autorização Apac, havia diferenças nas variáveis relativas à CID principal e ao sexo do paciente entre as bases.

Outra estratégia foi avaliar a base nacional do DATASUS de quimioterapia e radioterapia e verificar se 
um mesmo CNS codificado aparecia com registros de diferentes sexos e municípios do estabelecimento de saúde onde foi realizado o tratamento.

Todas as análises foram realizadas no software $R$ v.3.5.0, com a utilização do pacote tidyverse $e^{10}$. O projeto foi aprovado pelo Comitê de Ética em Pesquisa do INCA, sob o número CAAE: 77845317.9.0000.5274.

\section{RESULTADO}

Após o relacionamento determinístico, obtiveram-se 298.028 registros de Apac, correspondendo a 79.228 CNS na base hospitalar (CNS) e 80.983 CNS na base do DATASUS (CNS codificados). Os registros foram agrupados segundo as modalidades terapêuticas: quimioterapia, radioterapia e quimioterapia concomitante à radioterapia (Tabela 1).

Tabela 1. Total de registros nos bancos de dados após o relacionamento determinístico da base hospitalar identificada e o banco DATASUS, 2010 a 2016

\begin{tabular}{c|c|c|c}
\hline Bancos & $\begin{array}{c}\text { Autorizações de } \\
\text { Procedimentos } \\
\text { de Alta } \\
\text { Complexidade }\end{array}$ & $\begin{array}{c}\text { Cartão } \\
\text { Nacional } \\
\text { de Saúde }\end{array}$ & $\begin{array}{c}\text { Cartão } \\
\text { Nacional } \\
\text { de Saúde } \\
\text { Codificado }\end{array}$ \\
\hline $\begin{array}{c}\text { Quimioterapia } \\
\text { Radioterapia }\end{array}$ & 24.178 & 19.341 & 19.447 \\
$\begin{array}{c}\text { Quimioterapia } \\
+\end{array}$ & 149.014 & 34.773 & 35.706 \\
\begin{tabular}{c} 
Radioterapia \\
\hline
\end{tabular}
\end{tabular}

Fonte: Sistema de Informação Ambulatorial - SIA/SUS. Base de dados do INCA.
No banco de dados de radioterapia, foram identificados 106 CNS com mais de uma codificação, o que representa $0,55 \%$ dos cartôes (106/19.341). No banco de quimioterapia, essa proporção foi de 2,83\% (712/25.114). No banco combinado (quimioterapia + radioterapia), a proporção de cartóes com mais de um código associado foi de 2,67\% (928/34.773). Não foi identificado nenhum caso em que um mesmo código tenha sido utilizado para mais de um CNS.

Considerando que, para um mesmo CNS, foram atribuídas diferentes codificaçóes na base do DATASUS, utilizar a variável CNS codificada para representar um caso, em vez de utilizar o registro identificado do CNS, superestimou a contagem de $45,5 \%$ das 77 CID do banco de quimioterapia, e 20,2\% das 84 CID do banco de radioterapia. Para a quimioterapia, a superestimação variou de $0,16 \%$ a $5,88 \%$, enquanto, na radioterapia, foi de $0,17 \%$ a $3,07 \%$, dependendo da CID (Figura 1). A CID mais superestimada na quimioterapia foi neoplasia maligna da junção retossigmoide (C19) e na radioterapia, foi neoplasia maligna da vulva (C51).

Entre os registros relacionados pelo número da autorização, a CID da neoplasia informada diferiu em dois registros, entre a base hospitalar e a do DATASUS. Já a variável sexo diferiu em 99 registros, os quais corresponderam a $33 \mathrm{CNS}$.

Avaliando os dados nacionais do DATASUS, apenas no que diz respeito às Apac-tipo 1, o banco de quimioterapia apresentou, para o período em estudo, 1.221.899 CNS codificados distintos, dos quais, 13.837 $(1,13 \%)$ apareceram em mais de um município para
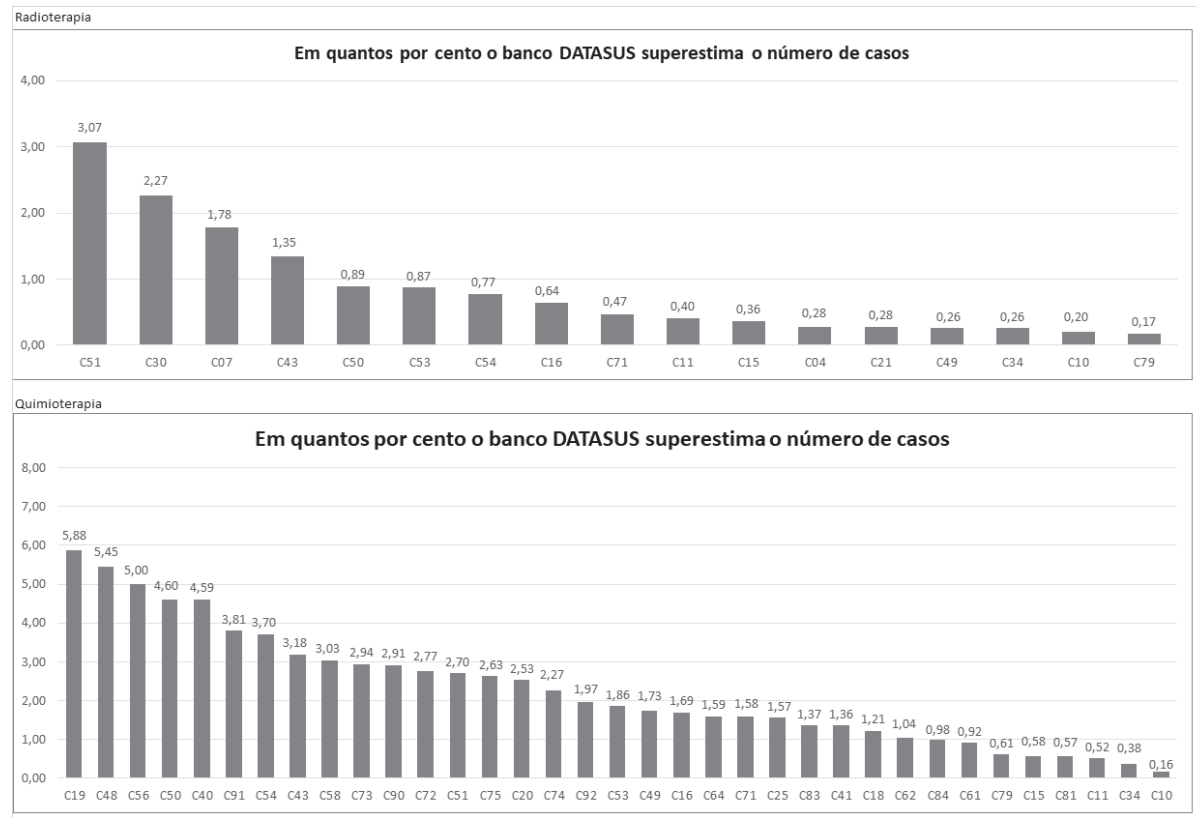

Figura 1. Percentual de superestimação do número de casos quando utilizado o CNS codificado da base do DATASUS, 2010 a 2016 
tratamento. O banco nacional de radioterapia possuía 700.368 CNS codificados e $9.790(1,40 \%)$ apareciam em estabelecimentos de saúde de diferentes municípios.

Ter um mesmo código associado a registros de diferentes sexos ocorreu em 329 casos no banco de radioterapia, representando $0,05 \%$ dos códigos de CNS e, no banco de quimioterapia, esse número chegou a 4.258 CNS codificados $(0,35 \%)$.

\section{DISCUSSÃO}

Há um debate sobre os métodos de relacionamento de bases de dados em saúde, considerando que podem ser determinísticas ou probabilísticas ${ }^{11-13}$. Na ausência de um identificador unívoco, quase sempre o rastreamento probabilístico possuí sensibilidade consideravelmente superior ao determinístico ${ }^{14}$. Diversos países, especialmente na Europa e na América do Norte, adotaram identificadores unívocos nos registros de saúde, seja usando números específicos do setor saúde ou outros já em uso para outras finalidades no país ${ }^{15}$. A existência de um identificador único permite relacionamento determinístico de diversos registros de um mesmo indivíduo de forma mais eficiente e confiável. Contudo, a implantaçáo desses identificadores é um grande desafio para países de média e baixa rendas ${ }^{16}$. O processo desenvolvido no presente artigo utilizou relacionamento determinístico para tentar estabelecer uma relação unívoca entre as bases de dados analisadas, permitindo discutir a confiabilidade da codificação do CNS como identificador único nas bases do DATASUS.

Este estudo identificou que, até o momento, a codificação do CNS utilizada nas bases de dados do DATASUS não permite garantir a identificação unívoca dos usuários do SUS, conforme estabelecido nas Portarias do Ministério da Saúde. Mesmo havendo uma correspondência majoritária entre as informaçôes das bases utilizadas, foram identificados problemas. Embora a proporção de CNS com mais de uma codificação tenha sido inferior a $3 \%$, esse tipo de erro pode produzir vieses importantes ao se tentar monitorar a assistência prestada ao usuário, utilizando-se um mesmo sistema de informação ou na combinação de informações provenientes de diferentes SIS.

Diversos estudos ${ }^{17-21}$ utilizam bases de acesso público do DATASUS e consideram a variável-chave codificada para definir "caso" e descrever o perfil da assistência. Outros estudos ${ }^{22-26}$ utilizam bases de dados identificadas; todavia, deparam-se com a dificuldade de obter tais bases. Dessa forma, a disponibilizaçáo de base de dados codificados é uma alternativa para realização das pesquisas, dispensando a cessão da base identificada nominalmente e assegurando a confidencialidade dos dados.
A utilização de bases codificadas, com os problemas identificados, pode gerar resultados distorcidos, uma vez que a não unicidade das codificações de CNS, além de não permitir o completo acompanhamento do indivíduo pelo sistema de saúde, infla os quantitativos de casos. Observou-se que as CID mais superestimadas não corresponderam às neoplasias mais frequentes no Rio de Janeiro/Brasil, descartando uma possível explicaçáo de um número maior de diferenças vinculado a maior ocorrência da neoplasia.

Outro problema identificado foi a ocorrência de registro na variável "sexo masculino" em uma base e "feminino" em outra, quando a variável cartão SUS correspondeu à variável codificada. Situação semelhante ocorreu para a variável de codificaçáo da neoplasia - CID. É possível que a diferença observada em relação à variável sexo seja em consequência de um ajuste na base nacional após a crítica realizada pelo DATASUS ${ }^{3,5}$. Todavia, essa adequação não explica a diferença observada no registro da CID.

Os casos em que um mesmo CNS codificado apareceu em estabelecimentos de saúde de diferentes municípios, embora possível, não nos permite afirmar que corresponda ao percurso terapêutico realizado pelo usuário, uma vez que o estudo mostrou que um mesmo código de CNS foi utilizado para diferentes indivíduos.

A principal limitação deste estudo refere-se à sua abrangência, uma vez que foram utilizados dados de um único hospital. Mesmo que esse hospital apresente maior produçáo local nessa área e seja referência para todo o Estado, faz-se necessária a ampliação dessa investigação para verificar se os resultados obtidos se mantêm para diferentes hospitais.

\section{CONCLUSÃO}

A codificação do CNS em todos os sistemas de informação é imperativa para garantir a privacidade dos dados dos usuários do SUS. Contudo, é necessário que haja a unicidade dessa codificação. Assim, será possível verificar os intervalos entre os procedimentos previstos nas linhas de cuidado, estimar custos de tratamento, identificar possível entraves ao acesso e planejar a organização da rede de atenção à saúde com vistas a atender aos princípios fundamentais do SUS. A qualificação dessa informação é estratégica para a gestão da assistência no SUS.

\section{CONTRIBUIÇÕES}

Adriana Tavares de Moraes Atty e Jeane Glaucia Tomazelli contribuíram com a concepção, delineamento do estudo e obtenção dos dados. Jeane Glaucia Tomazelli, 
Adriana Tavares de Moraes Atty, Maria Beatriz Kneipp Dias, Caroline Madalena Ribeiro e Neilane Bertoni contribuíram na análise, interpretação dos dados e redação do texto. Jeane Glaucia Tomazelli, Adriana Tavares de Moraes Atty, Maria Beatriz Kneipp Dias, Caroline Madalena Ribeiro, Arn Migowski e Neilane Bertoni participaram da revisão crítica do conteúdo intelectual do manuscrito e aprovaram a versão final.

\section{DECLARAÇÃO DE CONFLITO DE INTERESSES}

Nada a declarar.

\section{FONTES DE FINANCIAMENTO}

Não há.

\section{REFERÊNCIAS}

1. Ministério da Saúde (BR), Secretaria de Gestão de Investimentos. $\mathrm{O}$ cartáo nacional de saúde: instrumento para um novo modelo de atenção. Rev Saúde Pública. 2000;34(5):561-4. http://dx.doi.org/10.1590/S003489102000000500020

2. Ministério da Saúde (BR); Organização Pan-Americana da Saúde; Fundação Oswaldo Cruz. A experiência brasileira em sistemas de informação em saúde. Vol. 1, Produção e disseminação de informaçôes sobre saúde no Brasil. Brasília, DF: Ministério da Saúde; 2009. (Série B; Textos Básicos de Saúde). [Cap. 3], Sistema de Informaçóes Hospitalares do SUS - SIH-SUS49; p. 49.

3. Ministério da Saúde (BR). Portaria no 940, de 28 de abril de 2011. Regulamenta o Sistema Cartão Nacional de Saúde (Sistema Cartão). Diário Oficial da Uniáo, Brasília, DF; 2011 maio 2. Seção I, p. 58.

4. Ministério da Saúde (BR). Portaria no 1.560, de 29 de agosto de 2002. Institui o Cartão Nacional de Saúde Cartão SUS e da outras providências. [Ementa elaborada pela CDI/MS]. Diário Oficial da União, Brasília, DF; 2002 set. 13. Seção I, p. 42.

5. Ministério da Saúde (BR). Portaria $n^{\circ} 174$, de 14 de maio de 2004. Determina que os procedimentos ambulatoriais e hospitalares que exigem autorização prévia sejam acompanhados, obrigatoriamente, $\mathrm{da}$ identificação dos usuários por meio do número do Cartáo Nacional de Saúde, conforme cronograma constante do anexo desta portaria. [Ementa elaborada pela biblioteca/MS]. Diário Oficial da União, Brasília, DF; 2004 maio 17. Seção I, p. 62.

6. Ministério da Saúde (BR). Portaria no 2.073, de 31 de agosto de 2011. Regulamenta o uso de padróes de interoperabilidade e informação em saúde para sistemas de informaçáo em saúde no âmbito do Sistema Único de Saúde, nos níveis Municipal, Distrital, Estadual e
Federal, e para os sistemas privados e do setor de saúde suplementar. Diário Oficial da União, Brasília, DF; 2011 set. 1. Seção I, p. 63.

7. Ministério da Saúde (BR). DATASUS: relatório executivo da gestão 2011-2014. Brasília, DF: DATASUS; 2015.

8. Ministério da Saúde (BR); Organização Pan-Americana da Saúde; Fundação Oswaldo Cruz. A experiência brasileira em sistemas de informação em saúde. Vol. 1, Produção e disseminação de informaçôes sobre saúde no Brasil. Brasília, DF: Ministério da Saúde; 2009. (Série B; Textos Básicos de Saúde). [Cap. 5], Departamento de Informática do SUS - DATASUS: a experiência de disseminação de informaçôes em saúde; p. 109.

9. Presidência da República (BR). Lei no 12.527 , de 18 de novembro de 2011. Regula o acesso a informaçôes previsto no inciso XXXIII do art. $5^{\circ}$, no inciso II do $\$ 3^{\circ}$ do art. 37 e no $₫ 2^{\circ}$ do art. 216 da Constituição Federal; altera a Lei $\mathrm{n}^{\circ}$ 8.112, de 11 de dezembro de 1990; revoga a Lei no 11.111 , de 5 de maio de 2005, e dispositivos da Lei no 8.159, de 8 de janeiro de 1991; e dá outras providências. Diário Oficial da União, Brasília, DF; 2011 nov. 18. Seção I, Edição extra, p. 1.

10. Wickham H, RStudio. Tidyverse: easily install and load the "Tidyverse" [Internet]. Version 1.2.1. Boston: Free Software Foundation, Inc.; 2017 - [cited 2018 Aug 3]. Available from: https://CRAN.R-project.org/ package $=$ tidyverse

11. Harron K, Dibben C, Boyd J, et al. Challenges in administrative data linkage for research. Big Data \& Society. 2017 Dec;4(2):205395171774567. doi: https:// doi.org/10.1177/2053951717745678

12. Coeli CM, Pinheiro RS, Camargo Jr. KR. Conquistas e desafios para o emprego das técnicas de record linkage na pesquisa e avaliaçáo em saúde no Brasil. Epidemiol Serv Saúde. 2015 out.;24(4):795-802. doi: https://doi. org/10.5123/S1679-49742015000400023

13. Jurczyk P, Lu JJ, Xiong L, et al. FRIL: a tool for comparative record linkage. AMIA Annu Symp Proc. 2008 Nov 6:440-4.

14. Zhu Y, Matsuyama Y, Ohashi Y, et al. When to conduct probabilistic linkage vs. deterministic linkage? A simulation study. J Biomed Inform. 2015 Aug;56:80-6. doi: https://doi.org/10.1016/j.jbi.2015.05.012

15. Organisation for Economic Co-operation and Development. Strenghtening heatlh information infrastucture for health care quality governance: good practices, new opportunities and data privacy protection challenges. Paris: OECD Health Policy Studies, OECD Publishing; 2013. doi: http://dx.doi. org/10.1787/9789264193505-en

16. Joint United Nations Programme on HIV/AIDS. Considerations and guidance for countries adopting national health identifiers[Internet]. Geneva, Switzerland: UNAIDS; c2014. [cited 2019 Oct 10]. 112 p. Available 
from: https://www.unaids.org/sites/default/files/media_ asset/JC2640_nationalhealthidentifiers_en.pdf

17. Gadelha MIP, Costa MR, Almeida RT. Estadiamento de Tumores Malignos - análise e sugestóes a partir de dados da APAC. Rev Bras Cancerol. 2005;51(3):193-9.

18. Gomes Jr SCS, De Martino R, Almeida RT. Rotinas de integração das tabelas do sistema de autorização de procedimentos de alta complexidade em oncologia do Sistema Único de Saúde. Cad Saúde Coletiva. 2003;11(2):231-54.

19. Souza RC, Freire SM. Integração de dados ambulatoriais de quimioterapia e radioterapia registrados nas bases de dados do SUS. In: 24th Congresso Brasileiro de Engenharia Biomédica; 2014 out. 13-17. Uberlândia, MG. Rio de Janeiro: Sociedade Brasileira de Engenharia Biomédica; 2014. p. 1904-07.

20. Atty ATM. O câncer de boca no Estado do Rio de Janeiro à luz dos sistemas de informação. [dissertação]. Rio de Janeiro: Escola Nacional de Saúde Pública Sergio Arouca, Fiocruz; 2012.

21. Atty ATM, Tomazelli JG, Dias, MBK. Análise exploratória das informaçôes sobre estadiamento nas autorizaçôes de procedimentos de alta complexidade no Brasil e regióes no período 2010-2014. Rev Bras Cancerol. 2017;63(4):257-64. doi: https://doi. org/10.32635/2176-9745.RBC.2017v63n4.126

22. Teixeira CLS, Bloch KV, Klein CH, et al. Método de relacionamento de bancos de dados do Sistema de Informações sobre Mortalidade (SIM) e das autorizações de internação hospitalar (BDAIH) no Sistema Único de Saúde (SUS), na investigação de óbitos de causa mal-definida no Estado do Rio de Janeiro, Brasil, 1998. Epidemiol Serv Saúde. 2006;15(1):47-57. doi: http:// dx.doi.org/10.5123/S1679-49742006000100004.

23. Suzuki KMF. O uso de método de relacionamento de dados (record linkage) para integração de informação em sistemas heterogêneos de saúde: estudo de aplicabilidade entre níveis primário e terciário. [Tese]. Ribeirão Preto, SP: Universidade de São Paulo, Faculdade de Medicina de Ribeirão Preto; 2012.

24. Girianelli VR. Fatores Associados ao Risco de Progressão para Câncer do Colo do Útero ou suas Lesóes Precursoras em Mulheres com Exame de Papanicolaou Negativo: um estudo de três anos de seguimento. [Tese]. Rio de Janeiro: Instituto Nacional de Câncer; 2008.

25. Cabral MDB. Proposta de relacionamento probabilístico dos registros da base de dados do programa de rastreamento do câncer do colo do útero. [Tese]. Rio de Janeiro: Universidade Federal do Rio de Janeiro; 2010.

26. Tomazelli JG, Girianelli VR, Silva GA. Mulheres rastreadas para câncer de mama: acompanhamento por meio dos Sistemas de Informação em Saúde. Epidemiol
Serv Saúde. 2018;27(3): e2017445. doi: http://dx.doi. org/10.5123/s1679-49742018000300005

Recebido em 24/7/2019 Aprovado em 11/10/2019 